\title{
Excitons in Boron Nitride Nanotubes: Dimensionality Effects
}

\author{
Ludger Wirtz, ${ }^{1,2}$ Andrea Marini, ${ }^{3,2}$ and Angel Rubio ${ }^{4,5,6,2}$ \\ ${ }^{1}$ Institute for Electronics, Microelectronics, and Nanotechnology (IEMN), CNRS-UMR 8520, \\ B.P. 60069, 59652 Villeneuve d'Ascq Cedex, France \\ ${ }^{2}$ European Theoretical Spectroscopy Facility (ETSF), 20018 San Sebastián, Spain \\ ${ }^{3}$ Istituto Nazionale per la Fisica della Materia e Dipartimento di Fisica dell'Universitá di Roma "Tor Vergata", \\ Via della Ricerca Scientifica, I-00133 Roma, Italy \\ ${ }^{4}$ Donostia International Physics Center (DIPC), 20018 Donostia-San Sebastián, Spain \\ ${ }^{5}$ Department of Material Physics, UPV/EHU and Centro Mixto CSIC-UPV, 20018 San Sebastián, Spain \\ ${ }^{6}$ Institut für Theoretische Physik, Freie Universität Berlin, Arnimallee 14, D-14195 Berlin, Germany
}

(Received 29 August 2005; published 30 March 2006)

\begin{abstract}
We show that the optical absorption spectra of boron nitride $(\mathrm{BN})$ nanotubes are dominated by strongly bound excitons. Our first-principles calculations indicate that the binding energy for the first and dominant excitonic peak depends sensitively on the dimensionality of the system, varying from $0.7 \mathrm{eV}$ in bulk hexagonal $\mathrm{BN}$ via $2.1 \mathrm{eV}$ in the single sheet of $\mathrm{BN}$ to more than $3 \mathrm{eV}$ in the hypothetical $(2,2)$ tube. The strongly localized nature of this exciton dictates the fast convergence of its binding energy with increasing tube diameter towards the sheet value. The absolute position of the first excitonic peak is almost independent of the tube radius and system dimensionality. This provides an explanation for the observed "optical gap" constancy for different tubes and bulk hexagonal BN.
\end{abstract}

PACS numbers: 81.07.De, 61.46.-w, 71.35.-y

In complete analogy to carbon nanotubes, boron nitride (BN) nanotubes $[1,2]$ can be thought of as cylinders that are obtained when a single sheet of hexagonal $\mathrm{BN}(\mathrm{hBN})$ is rolled onto itself. Since hexagonal BN is a large band-gap insulator [3], the band gap of BN tubes is similarly large, independent of their radius and chirality. The detailed knowledge of the optical properties of BN tubes is indispensable for their characterization and may help to guide their use as nanoelectronic devices. Example BN nanotubes have been used to build a field effect transistor [4]. Furthermore, experiments on ultraviolet luminescence [5] of bulk hexagonal BN suggest to explore the use of BN nanotubes as ultraviolet light sources. In this context, it is crucial to know about possible excitonic states whose importance has been recently shown for the optical spectra of carbon nanotubes [6,7]. For the wide band-gap BN tubes, we expect even stronger excitonic effects.

Very recently, two experimental studies of the optical properties of $\mathrm{BN}$ nanotubes have appeared in this journal which strongly contradict each other. Both studies compare their spectra to the one of bulk BN which has its first absorption peak at $6.1 \mathrm{eV}$ and an onset of absorption at about $5.8 \mathrm{eV}$. Lauret et al. [8] have measured two additional peaks in the optical absorption spectra of BN tubes at 4.45 and $5.5 \mathrm{eV}$. The lower of these two peaks was interpreted as a due to a bound exciton. Arenal et al. [9], on the contrary, have measured the electron-energy loss spectra (EELS) of isolated BN tubes and obtained a constant "optical gap" of $5.8 \mathrm{eV}$ for bulk BN and different single and multiwall tubes. For a proper interpretation of the spectra, one has to take into account that already the absorption peak of bulk hexagonal $\mathrm{BN}$ at $6.1 \mathrm{eV}$ is due to a strongly bound Frenkel exciton [10,11]. The question to be asked is therefore: how does the binding energy of this exciton change as we compare the quasi-two-dimensional $\mathrm{BN}$ sheet and the quasi-1D BN nanotubes with the 3D bulk BN? Furthermore: up to which diameter do tubes exhibit one-dimensional excitonic effects? We show in this Letter that the excitonic binding energy increases strongly with lower dimensionality. At the same time, however, the quasiparticle gap strongly increases such that the absolute position of the first (excitonic) absorption peak remains almost constant in agreement with Ref. [9].

So far, the optical properties of BN nanotubes have only been calculated $[12,13]$ on the level of the random-phase approximation (RPA), i.e., in the picture of independentparticle excitations. Here, we use the methods of manybody perturbation theory to include electron-electron and electron-hole effects [14]. Our calculations of the optical absorption spectra proceed in three steps. We first calculate the wave functions of the valence band states and a large number of conduction band states using density functional theory (DFT) in the local-density approximation (LDA) [15-17]. In the second step, we use the $G W$ approximation $[14,18]$ to calculate the quasiparticle energies ("true" single-particle excitation energies). In the third step, effects of electron-hole attraction (excitonic effects) are included by solving the Bethe-Salpeter (BS) equation [14].

Calculation details: we use a trigonal array of tubes with minimum interwall distance of 20 a.u. in order to minimize intertube interaction and to simulate as closely as possible the properties of isolated tubes. The tubes are geometry optimized (forces on the atoms less than $5 \times 10^{-5}$ a.u.). In the $G W$ calculation [19] we perform a "semiself consistent" $\left(G W_{0}\right)$ calculation by updating the quasiparticle energies in $G$ (but not in $W$ ) until the resulting quasiparticle 
energies are converged [20]. For the optical absorption spectra of $(n, n)$ armchair tubes and $(n, 0)$ zigzag tubes with polarization along the tube axis, transitions between the highest $2 n$ valence bands (the $\pi$ bands) and the lowest $2 n$ conduction bands (the $\pi^{*}$ bands) are taken into account (the other transitions being dipole forbidden).

In Fig. 1, we investigate the influence of the supercell geometry on the excitonic binding energy and on the quasiparticle gap of the single sheet of hexagonal BN. The spectrum is dominated by the lowest bound exciton which collects most of the oscillator strength in the energy range between 0 and $8 \mathrm{eV}$ [see Fig. 2(b)]. The excitonic binding energy is measured as the distance between this peak and the onset of the continuum which is given by the direct quasiparticle gap between the $\pi$ and $\pi^{*}$ bands. With increasing intersheet distance, approaching the limit of a quasi-2D isolated sheet, the excitonic binding energy increases and converges towards the value of $2.1 \mathrm{eV}$ [as compared to the binding energy of $0.7 \mathrm{eV}$ that is found for the 3D bulk hexagonal $\mathrm{BN}$ [11] ]. This increase of the binding energy is due to two effects: (i) separating the layers, screening is reduced and becomes more anisotropic. In particular, for the perpendicular direction, it gets close to 1. The weaker screening leads thereby to an increase of the excitonic binding energy. (ii) An increased electron-hole overlap in the reduced dimensionality leads also to a stronger binding energy [in the purely $2 \mathrm{D}$ limit, the binding energy for a hydrogenic system is increased by a factor of 4 compared to the 3D case [21] ]. At the same time, reduced dimensionality and reduced screening lead to an increased electron-electron correlation and thereby to an increase of the quasiparticle gap [22]. Figure 1 demonstrates that the increase of the quasiparticle gap almost exactly cancels the increase of the binding energy. The position of the first absorption peak remains almost constant. What changes is the onset of the continuum. For the BN sheet, however, the absorption at the onset of the continuum is almost zero (also the higher excitonic peaks carry very low oscillator strength). The excitonic spectrum can therefore be calcu-

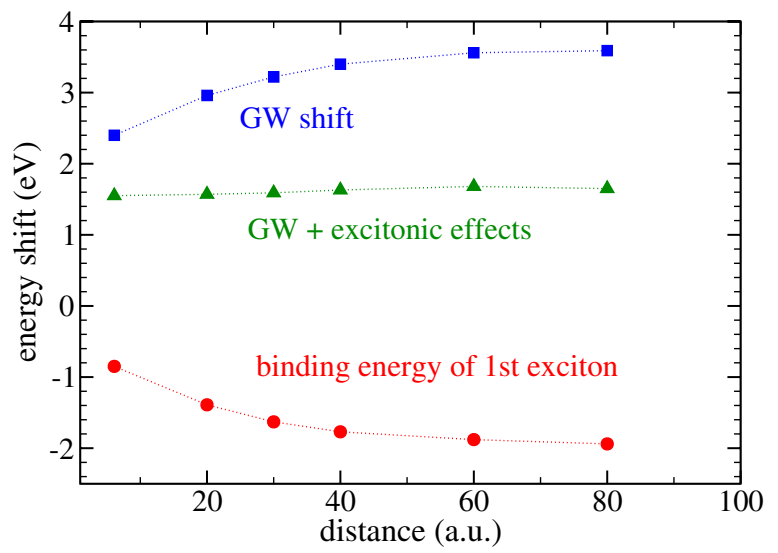

FIG. 1 (color online). Single sheet of hexagonal BN: dependence of the excitonic binding energy and the quasiparticle gap on the intersheet distance in a supercell geometry. lated to a good approximation already with an intersheet distance of 20 a.u. We made a similar series of calculations for the hypothetical $\mathrm{BN}(2,2)$ tube which has a diameter of $2.8 \AA$ and is close to being a 1D system. Again, as we increase the intertube distance, the increase of the quasiparticle gap almost cancels the increase of the excitonic binding energy. While the latter converges towards a value higher than $3 \mathrm{eV}$, the absolute position of the first absorption peak remains constant to within $0.2 \mathrm{eV}$. In the following, we present therefore calculations for different tubes in a supercell geometry with $20 \AA$ interwall distance. We remark that dimensionality effects would be more visible in other spectroscopic measurements such as photoemission spectroscopy, where we mainly map the quasiparticle spectra, and this (as the exciton binding itself) is sensitive to the change in screening going from the tube to the sheet to bulk hexagonal $\mathrm{BN}$. In particular, the quasiparticle band gap will vary strongly with dimensionality (opening as dimensionality reduces).

In Fig. 2 we present the spectra of bulk hexagonal BN, of the single sheet of hexagonal $\mathrm{BN}$, and of different $\mathrm{BN}$ nanotubes with diameters ranging from $2.8 \AA$ (for the purely hypothetical $\mathrm{BN}(2,2)$ tube) to $9.7 \AA$ (for the $\mathrm{BN}(7,7)$ tube) which is at the lower border of the range of experimentally produced tubes. The light polarization is set parallel to the planes or tube axis, respectively. On the left-hand side, we show the RPA spectra which are almost
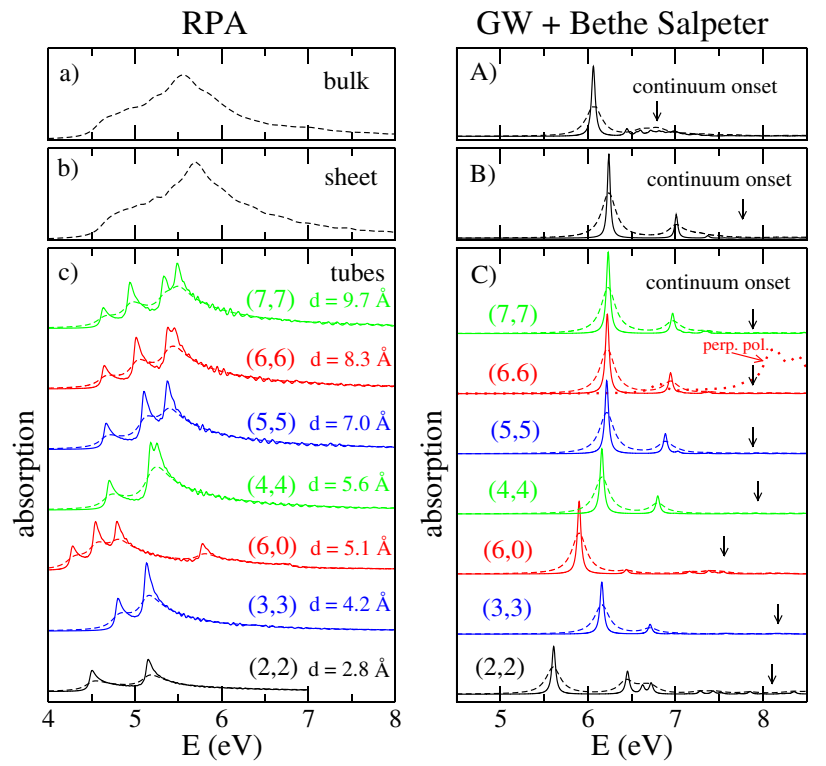

FIG. 2 (color online). Optical absorption of (a) hexagonal BN, (b) $\mathrm{BN}$ sheet, and (c) six different $\mathrm{BN}$ tubes with increasing diameter $d$. We compare the results of the $G W+$ Bethe-Salpeter approach (right-hand side) with the random-phase approximation (left-hand side). Solid lines are calculated with a Lorentzian broadening of $0.025 \mathrm{eV}$, dashed lines with a broadening of $0.1 \mathrm{eV}$ (for comparison with experimental data). The light polarization is parallel to the plane/tube axis, respectively [except for the dotted line in the $(6,6)$ case where the light polarization is perpendicular to the tube axis; see main text for discussion]. 
indistinguishable for the bulk and for the single sheet. The selection rules only allow transitions between the $\pi$ and $\pi^{*}$ bands (band 4 and 5 in the sheet). The band structure of the tubes can be constructed via the zone-folding procedure, i.e., by cutting the $2 \mathrm{D}$ band structure of the sheet along certain discrete lines that correspond to quantized wave vector along the circumferential direction. The RPA spectra of the tubes display therefore transitions at the same energies as in the sheet. [For a comparison of tube and sheet band-structures, see Refs. [1,3,13].] With increasing diameter, the shape of the tube spectra converges rapidly towards the sheet spectrum, in particular, if plotted with a Lorentzian broadening of $0.1 \mathrm{eV}$ (corresponding roughly to usual experimental values). A calculation with a fine broadening of $0.025 \mathrm{eV}$ (and a correspondingly fine sampling with $200 \mathbf{k}$ points in the first Brillouin zone) reveals additional fine structure below $5.5 \mathrm{eV}$. This structure is due to the van Hove singularities in the one-dimensional density of states. For tubes with larger radii, the density of the fine-structure peaks increases and the RPA spectrum approaches that of the $2 \mathrm{D}$ sheet. The onset of absorption is constantly at $4.7 \pm 0.1 \mathrm{eV}$ for all tubes except for the $(2,2)$ and the $(6,0)$ tube (and other small diameter zigzag tubes) where the gap is lowered due to curvature effects [1].

While the RPA spectra are due to a continuum of interband transitions, the BS $+G W$ optical spectra on the righthand side of Fig. 2 are dominated by discrete excitonic peaks where the first peak comprises most of the oscillator strength. For bulk hexagonal BN, we have shown [11] that the broadened excitonic spectrum properly reproduces the experimental spectral shape [23]. The sheet spectrum contains three bound excitonic peaks of rapidly decreasing intensity and absorption at the onset of the continuum is reduced to almost zero. As explained above, the stronger binding energy of the first bound exciton is almost compensated by an increase of the quasiparticle gap due to the reduced dimensionality. The same holds for the tube spectra: except for the three smallest tubes, the position of the first and dominant excitonic peak remains constant. With increasing tube diameter, the spectrum rapidly converges towards the three-peak spectrum of the flat sheet. Also, the onset of the continuum converges towards the value in the sheet (note that we compare here the values for a supercell geometry with intersheet or intertube distance of 20 a.u.). The rapid convergence of the excitonic peaks is an indication for a strong confinement of the exciton wave function. Plotting the wave function, we have verified that this Frenkel type exciton is confined to within a few interatomic distances for either tubes, sheet or bulk hexagonal BN [see also the plot for an exciton in bulk hexagonal $\mathrm{BN}$ in Ref. [10]]. With increasing tube diameter, the excitons only "see" a locally flat environment which explains the rapid convergence towards the sheet spectrum. The strongly localized nature of the exciton in BN structures makes the appearance of one-dimensional confinement effects very restricted to small diameter tubes, i.e., tubes for which the extension of the excitonic wave function is comparable to the nanotube circumference. As the experimental tubes have diameters around $1.4 \mathrm{~nm}$, the $1 \mathrm{D}$ nature of the tubes cannot be observed and only the 2D nature of the local exciton environment (tube surface) controls the optical activity. Many-body effects in carbon nanotubes have been found to be quite different [6,7]: binding energies and quasiparticle shifts are much smaller, and the extension of the excitonic wave function (several $\mathrm{nm}$ ) is larger than the typical tube circumference. Thus, excitonic binding energies strongly vary with the diameter. Contrary to the case of BN tubes, where we quickly reach the twodimensional limit of the flat sheet, excitons in carbon nanotubes remain one-dimensional objects, i.e., squeezed in the circumferential direction.

For the $(6,6)$ tube, we display in Fig. 2(c) also the spectrum for light polarization perpendicular to the tube axis. The spectrum exhibits a major excitonic peak that lies slightly below the second excitonic peak obtained for light polarized along the tube axis. Note that the sheet is completely transparent up to $9 \mathrm{eV}$ for light polarized perpendicular to the plane.

We compare our results now to two recent contradictory measurements of the optical properties of BN nanotubes [8,9]: in the EELS experiment of Arenal et al. [9], the electron beam passes the tube in the tangential direction. A quantitative explanation of the spectral shape would require the calculation of the imaginary part of the polarizability $\alpha_{m, k}$, where $m$ is the index for the multipole expansion in circumferential direction and $k$ is the wave vector of the Fourier expansion along the tube axis [24]. However, the dipolar contribution $(m=0)$ in the limit $k \rightarrow$ 0 , calculated in this Letter, is the dominant part in the expansion of $\alpha$. The constancy of the first excitonic peak in Fig. 2 explains why the "optical gap" observed in Fig. 2 of Ref. [9] is always $5.8 \mathrm{eV}$, independently if they measure multiwall tubes, or single-wall tubes of different diameters. The shoulder on the right-hand side of the main peak in Fig. 2c of Ref. [9] is the effect of the 2nd excitonic peak. Figure 2 demonstrates that the second (and less intense) excitonic peak starts to form a shoulder of the first peak as we plot the spectra with a stronger broadening. Our calculations also reproduce the finding of Ref. [9] that the dominant peak is higher in energy for the tubes [their Figs. 2(a)-(c)] than for bulk BN [their Fig. 2(d)] [25].

We note that there is a chirality dependence of the optical spectra but it is only visible for the smallest diameter tubes. The spectra of the armchair tubes converge much faster to the $2 \mathrm{D}$ case than the ones of the zigzag tubes. However, as experimental tube diameters [9] are much larger than the $5.1 \AA$ of the $(6,0)$ zigzag tube that is presented in this Letter, we expect the chiral dependence to be marginal. [Results similar to the $(6,0)$ tube are presented in Ref. [26] for the $(8,0)$ tube]. Our calculations show that the explanation of Ref. [8] for the two peaks at 4.45 and $5.5 \mathrm{eV}$ in their absorption spectra of a sample containing $\mathrm{BN}$ tubes does not hold: the peaks are neither 
due to additional van Hove singularities (since the spectra are entirely dominated by discrete excitonic peaks) nor can they be explained by an increased excitonic binding energy (which is canceled by an increased quasiparticle gap).

So far, we have concentrated on singlet active excitons. For bulk hexagonal BN [11], we have shown previously that there is a dark singlet exciton and two triplet excitons below the first optically active exciton. For the single sheet (and light polarization parallel to the plane), we find that the lowest optically active exciton is doubly degenerate. There is no dark singlet exciton below, but a doubly degenerate triplet exciton at $0.1 \mathrm{eV}$ lower energy. For the $(6,6)$ tube (and light polarization parallel to the tube axis), we find that the degeneracy of the singlet exciton is lifted, leading to a dark singlet exciton slightly $(0.01 \mathrm{eV})$ below the optically active singlet exciton. The degeneracy of the triplet exciton is lifted as well: the two triplet excitons are $0.1 \mathrm{eV}$ and $0.08 \mathrm{eV}$ lower in energy than the optically active singlet exciton. Similar results hold for the $(5,5)$ and the $(7,7)$ tubes. A recent study for $\mathrm{C}$ tubes [27] has shown that the room temperature luminescence is enhanced once the complete series of active and dark excitons is taken into account. This would hold also in the present case. Furthermore, due to the minor differences in the optical spectra of tubes and bulk BN we expect the BN tubes to exhibit a strong ultraviolet lasing behavior as already observed for bulk BN [5]. The fact that this luminescence response would be rather insensitive to tube diameter and chirality makes the BN tubes ideal candidates for optical devices in the UV regime as the carbon nanotubes are in the infrared regime [28]. The photoluminescence quantum yield of BN tubes should surpass the efficiency of carbon [5].

We acknowledge helpful discussions with D. Varsano, V. Olevano, and L. Reining. The work was supported by the EU network of excellence NANOQUANTA (NMP4CT-2004-500198) and the French GDR "nanotubes." Calculations were performed at IDRIS (Project No. 51827) and CEPBA. A.R. acknowledges the Humboldt Foundation.

[1] A. Rubio, J. L. Corkill, and M. L. Cohen, Phys. Rev. B 49, R5081 (1994); X. Blase, A. Rubio, S. G. Louie, and M. L. Cohen, Europhys. Lett. 28, 335 (1994).

[2] N. G. Chopra et al., Science 269, 966 (1995).

[3] X. Blase, A. Rubio, S. G. Louie, and M. L. Cohen, Phys. Rev. B 51, 6868 (1995).

[4] M. Radosavljević et al., Appl. Phys. Lett. 82, 4131 (2003).

[5] K. Watanabe, T. Taniguchi, and H. Kanda, Nat. Mater. 3, 404 (2004).

[6] C. D. Spataru, S. Ismail-Beigi, L. X. Benedict, and S. G. Louie, Phys. Rev. Lett. 92, 077402 (2004); Appl. Phys. A 78, 1129 (2004).
[7] E. Chang, G. Bussi, A. Ruini, and E. Molinari, Phys. Rev. Lett. 92, 196401 (2004).

[8] J.S. Lauret et al., Phys. Rev. Lett. 94, 037405 (2005).

[9] R. Arenal, O. Stéphan, M. Kociak, D. Taverna, A. Loiseau, and C. Colliex, Phys. Rev. Lett. 95, 127601 (2005).

[10] B. Arnaud, S. Lebègue, P. Rabiller, and M. Alouani, Phys. Rev. Lett. 96, 026402 (2006).

[11] L. Wirtz, A. Marini, M. Grüning, and A. Rubio (to be published); cond-mat/0508421.

[12] A. G. Marinopoulos, L. Wirtz, A. Marini, V. Olevano, A. Rubio, and L. Reining, Appl. Phys. A 78, 1157 (2004).

[13] G. Y. Guo and J. C. Lin, Phys. Rev. B 71, 165402 (2005).

[14] For a recent review see, e.g., G. Onida, L. Reining, and A. Rubio, Rev. Mod. Phys. 74, 601 (2002).

[15] W. Kohn and L. J. Sham, Phys. Rev. 140, A1133 (1965).

[16] We use the code ABINIT: X. Gonze et al., Comput. Mater. Sci. 25, 478 (2002).

[17] We used Trouiller-Martins pseudopotentials in the Kleinman-Bylander form with cutoff radius of 1.59 a.u. for B and 1.50 a.u. for N. An energy cutoff of 25 hartree is used in the plane-wave expansion of the wave functions.

[18] M. S. Hybertsen and S. G. Louie, Phys. Rev. B 34, 5390 (1986).

[19] We use the computer code SELF, http://www.fisica. uniroma2.it/ self/, written by A. Marini.

[20] This procedure yields a quasiparticle band gap for the single sheet and for the tubes that is about $0.25 \mathrm{eV}$ higher than the one obtained on the nonself consistent $\left(G_{0} W_{0}\right)$ level. Still, for bulk hexagonal BN we have shown [11] that this $G W_{0}$ calculation starting from LDA wave functions yields a quasiparticle band gap that is too low by $0.65 \mathrm{eV}$. In the present work, we assume that the same underestimation of the LDA $G W_{0}$ band gap holds also for the single sheet and for the tubes. Consequently, we add a $0.65 \mathrm{eV}$ "scissor" shift to the calculated $G W_{0}$ band structure. The assumption that this shift is constant for bulk, sheet, and tubes is certainly only a zero order approximation. Closer comparison with the measured "optical gap" data of BN tubes in Fig. 2 of Ref. [9] indicates that this shift could be up to $0.35 \mathrm{eV}$ higher for the tubes than for the bulk.

[21] M. Shinada and S. Sugano, J. Phys. Soc. Jpn. 21, 1936 (1966); T. G. Pedersen, Phys. Rev. B 67, 073401 (2003).

[22] C. Delerue, G. Allan, and M. Lannoo, Phys. Rev. Lett. 90, 076803 (2003).

[23] C. Tarrio and S. E. Schnatterly, Phys. Rev. B 40, 7852 (1989).

[24] D. Taverna, M. Kociak, V. Charbois, and L. Henrard, Phys. Rev. B 66, 235419 (2002).

[25] This energy shift is $0.55 \mathrm{eV}$ in Ref. [9] while we obtain in Fig. 2 a shift of $0.2 \mathrm{eV}$. This indicates that the underestimation of the band gap by the $G W$ approximation is indeed slightly higher for the tubes than for bulk BN [see Ref. [20] ].

[26] C.-H. Park, C. D. Spataru, and S. G. Louie, this issue, Phys. Rev. Lett. 96, 126105 (2006).

[27] C. D. Spataru, S. Ismael-Beiji, R. B. Capaz, and S. G. Louie, Phys. Rev. Lett. 95, 247402 (2005).

[28] J.A. Misewich, R. Martel, Ph. Avouris, J.C. Tsang, S. Heinze, and J. Tersoff, Science 300, 783 (2003). 